\title{
Symptomatic improvement after radiofrequency catheter ablation for typical atrial flutter
}

\author{
P A O’Callaghan, M Meara, E Kongsgaard, J Poloniecki, L Luddington, J Foran, \\ A J Camm, E Rowland, D E Ward
}

\begin{abstract}
Objective-To assess the changes in quality of life, arrhythmia symptoms, and hospital resource utilisation following catheter ablation of typical atrial flutter.

Design-Patient questionnaire to compare the time interval following ablation with a similar time interval before ablation.

Setting-Tertiary referral centre.

Patients-63 consecutive patients were studied. Four patients subsequently underwent an ablate and pace procedure, two died of co-morbid illnesses, and two were lost to follow up. The remaining 55 patients form the basis of the report.

Results-Patients were followed for a mean (SD) of 12 (9.5) months. Atrial flutter ablation resulted in an improvement in quality of life $(3.8 v 2.5, \mathrm{p}<0.001)$ and reductions in symptom frequency score $(2.0 v 3.5, \mathrm{p}<0.001)$ and symptom severity score $(2.0 v 3.8, \mathrm{p}<0.001)$ compared with preablation values. There was a reduction in the number of patients visiting accident and emergency departments $(11 \% v 53 \%, \mathrm{p}<0.001)$, requiring cardioversion $(7 \% v 51 \%$, $\mathrm{p}<0.001)$, or being admitted to hospital for a rhythm problem $(11 \% v 56 \%, \mathrm{p}<0.001)$. Subgroup analysis confirmed that patients with atrial flutter and concomitant atrial fibrillation before ablation and those with atrial flutter alone both derived significant benefit from atrial flutter ablation. Patients with concomitant atrial fibrillation had an improvement in quality of life (3.5 $v 2.5, \mathrm{p}<0.001)$ and reductions in symptom frequency score $(2.3 v 3.5, \mathrm{p}<0.001)$ and symptom severity score $(2.2 v 3.7, \mathrm{p}<0.001)$ compared with preablation values.

Conclusions-Ablation of atrial flutter is recommended both in patients with atrial flutter alone and in those with concomitant atrial fibrillation.

(Heart 2001;86:167-171)
\end{abstract}

Keywords: atrial flutter; radiofrequency ablation; quality of life

Typical atrial flutter is caused by a right atrial macroreentry circuit which incorporates, as a critical component, an isthmus of atrial tissue surrounded by the tricuspid annulus anteriorly, the inferior vena cava posteriorly, and the coronary sinus and Eustachian ridge septally. ${ }^{1-3}$ Radiofrequency catheter ablation causing bidirectional isthmus conduction block results in a low recurrence rate of atrial flutter..$^{4-8}$ However, in contrast to patients with atrioventricular nodal re-entry tachycardia (AVNRT) or atrioventricular re-entry tachycardia (AVRT), atrial flutter does not typically occur in isolation. A high proportion of patients with atrial flutter also have documented atrial fibrillation or underlying structural heart disease. Although atrial flutter can be effectively ablated, few studies have assessed the effects of atrial flutter ablation on patients' quality of life..$^{9-11}$ We report the changes in symptom frequency, symptom severity, perceived quality of life, and hospital resource utilisation in a consecutive series of patients who underwent catheter ablation of typical atrial flutter at our institution.

\section{Methods}

All patients who had catheter ablation of typical atrial flutter performed between 1997 and 1999 were studied. Each patient's medical record was reviewed by one of the investigators. The results of electrocardiographic recordings (ECGs, 24 hour Holter monitors, and event monitors), echocardiograms, and diagnostic cardiac catheterisation were employed to determine the baseline characteristics.

Radiofrequency catheter ablation was performed in a unipolar temperature guided mode (target temperature $60-65^{\circ} \mathrm{C}$ ) using either a $4 \mathrm{~mm}$ or an $8 \mathrm{~mm}$ tip ablation electrode. The procedure end point was complete bidirectional isthmus conduction block. This was assessed using conventional electrophysiological methods. ${ }^{57}$

\section{LONG TERM FOLLOW UP AND PATIENT} QUESTIONNAIRE

Each patient was contacted and asked to complete a detailed questionnaire. This was a modified version of the questionnaire used by Fitzpatrick and colleagues. ${ }^{12}$ Patients were asked if they had had any attack of their rhythm problem since their ablation. Patients compared the time interval following ablation with a similar time interval before ablation. In the assessment of general quality of life, scoring was: 1 , very poor; 2 , poor; 3 , good; 4 , very good; 5, excellent. Symptom frequency was scored as: 1 , never; 2 , infrequent; 3 , sometimes; 4 , very frequent. Symptom severity was scored as: 1 , none; 2 , mild; 3 , moderate; 4 , severe; 5 , very severe. Patients also scored their general quality of life since ablation: 1 , much worse; 2 , worse, 3 , unchanged; 4 , better; 5 , much better. In addition they were asked if they had 
Table 1 Baseline characteristics

\begin{tabular}{ll}
\hline Patients (n) & 55 \\
Age (years) & \\
$\quad$ Mean (SD) & $57.5(12)$ \\
Range & 22 to 77 \\
Atrial flutter (n (\%)) & \\
$\quad$ Paroxysmal & $34(62 \%)$ \\
$\quad$ Persistent & $21(38 \%)$ \\
Atrial fibrillation (n (\%)) & $29(53 \%)$ \\
Structural heart disease (n (\%)) & $19(35 \%)$ \\
Antiarrhythmic drug trials & 2 \\
$\quad$ Median & 2 \\
Range & 0 to 5 \\
Amiodarone & $27(49 \%)$ \\
No drug trial & $2(4 \%)$ \\
\hline
\end{tabular}

Table 2 Acute results

\begin{tabular}{ll}
\hline Procedure time (min) & \\
Mean (SD) & $124(45)$ \\
Range & $60-240$ \\
Fluroscopy time (min) & \\
Mean (SD) & $34(17)$ \\
Range & $12-78$ \\
Isthmus block (n (\%)) & $45(83 \%)$ \\
Bidirectional block & $3(6 \%)$ \\
Unidirectional block & $6(11 \%)$ \\
No complete block & $1(1.8 \%)$ \\
Complications (n (\%)) & $1(1.8 \%)$ \\
Complete heart block/pacemaker & $1(1.8 \%)$ \\
Deep vein thrombosis & $1(1.8 \%)$ \\
Worsening heart failure & \\
Skin burn & \\
\hline One data point missing. &
\end{tabular}

attended an accident and emergency department, had an electrical cardioversion, or had been admitted to hospital because of a rhythm problem, and to compare the time interval following ablation with a similar time interval before ablation. Any discrepancy between the questionnaire and the patient's medical record was clarified with the patient. Patients who experience palpitations following ablation at our institution are monitored with a 24 hour Holter monitor or an event recorder, or both.

STATISTICAL ANALYSIS

Results are expressed as mean (SD) or mean (SEM) as indicated. Within group comparisons were performed using the non-parametric sign test. Between group comparisons were performed using the Mann-Whitney U test. A two tailed probability value of $p<0.05$ was considered significant.

\section{Results}

Sixty three patients had catheter ablation of typical atrial flutter performed over the two year study period. Four of these patients

Table 3 Long term follow up results compared with preablation values

\begin{tabular}{|c|c|c|c|}
\hline & Preablation & Follow up & $\begin{array}{l}\text { p Value } \\
\text { (sign test) }\end{array}$ \\
\hline Atrial fibrillation (n (\%)) & $29(53 \%)$ & $15(28 \%)$ & 0.002 \\
\hline \multicolumn{4}{|l|}{ Antiarrhythmic drugs (n (\%)) } \\
\hline Any antiarrhythmic drug & $53(96 \%)$ & $27(49 \%)$ & $<0.001$ \\
\hline Amiodarone & $27(49 \%)$ & $9(16 \%)$ & $<0.001$ \\
\hline $\mathrm{A} \& \mathrm{E}$ visits $(\mathrm{n}(\%))^{\star}$ & $29(53 \%)$ & $5(11 \%)$ & $<0.001$ \\
\hline $\operatorname{DCCV}(\mathrm{n}(\%))^{\star}$ & $28(51 \%)$ & $4(7 \%)$ & $<0.001$ \\
\hline \multicolumn{4}{|l|}{ Hospital admissions } \\
\hline Any admission $(\mathrm{n}(\%)) \dagger$ & $31(56 \%)$ & $6(11 \%)$ & $<0.001$ \\
\hline Mean number of days/patient & 9.5 & 0.6 & $<0.001$ \\
\hline \multicolumn{4}{|c|}{ Patient questionnaire $(\text { mean }(\text { SEM }))^{\star}$} \\
\hline Quality of life score & $2.5(0.15)$ & $3.8(0.13)$ & $<0.001$ \\
\hline Symptom frequency score & $3.5(0.11)$ & $2.0(0.13)$ & $<0.001$ \\
\hline Symptom severity score & $3.8(0.15)$ & $2.0(0.15)$ & $<0.001$ \\
\hline
\end{tabular}

${ }^{\star}$ Comparison of the time interval following ablation with the same time interval before ablation. †Includes any hospital admission as a result of a rhythm problem.

$\mathrm{A} \& \mathrm{E}$, accident and emergency department; DCCV, direct current cardioversion. subsequently underwent atrioventricular node ablation and pacemaker implantation, two died of co-morbid illnesses (14 and 19 months after ablation), and two were lost to follow up. The remaining 55 patients form the basis of the study. Their baseline characteristics are shown in table 1 .

More than one third of the patients suffered from persistent atrial flutter, over half had documented atrial fibrillation before ablation, and $35 \%$ had underlying structural heart disease. Patients had failed a median of two antiarrhythmic drug trials, and nearly half the group had failed treatment with amiodarone before catheter ablation. The acute ablation results are shown in table 2. Complete bidirectional isthmus conduction block was confirmed in $83 \%$ of the patients.

ARRHYTHMIA BURDEN

Patients were followed for a mean (SD) of 12 (9.5) months. During follow up, four patients had electrocardiographically documented "atrial flutter". Three of these patients underwent a repeat electrophysiological study which confirmed bidirectional isthmus conduction block and a diagnosis of either atypical non-isthmus-dependent atrial flutter or atrial tachycardia was made. The fourth patient had frequent attacks of both paroxysmal atrial fibrillation and atrial flutter and did not undergo a further electrophysiological study. Five of the original 63 patients required cardioversion for atrial fibrillation during the ablation procedure, of whom three underwent atrioventricular node ablation and pacemaker implantation. The remaining two patients followed long term in this study had no recurrence of atrial fibrillation.

Long term follow up results are shown in table 3 . Twenty nine patients $(53 \%)$ had atrial fibrillation before ablation, compared with 15 patients $(28 \%)$ with atrial fibrillation during the follow up period $(\mathrm{p}<0.002)$. Eleven of the 29 patients with preablation atrial fibrillation (38\%) had a recurrence of atrial fibrillation, and four of 26 patients without preablation atrial fibrillation (15\%) developed atrial fibrillation during the follow up period $(\mathrm{p}<0.002)$. There was a significant reduction in antiarrhythmic drug use, visits to accident and emergency departments, and hospital admissions for rhythm problems following atrial flutter ablation. Half the patients (28 of 55) underwent cardioversion and one third (18 of $55)$ required repeated $(\geqslant 2)$ cardioversions before ablation. During a similar time interval following ablation, four patients (7\%) underwent cardioversion and only one (1.8\%) required repeated cardioversions.

\section{PATIENT QUESTIONNAIRE}

Twenty six of the 55 patients $(47 \%)$ reported that they had experienced an attack of their rhythm problem since the ablation procedure. The mean quality of life, symptom frequency score, and symptom severity score following ablation, compared with the scores for a similar time interval before ablation, are shown in table 3. There was a significant improvement in 

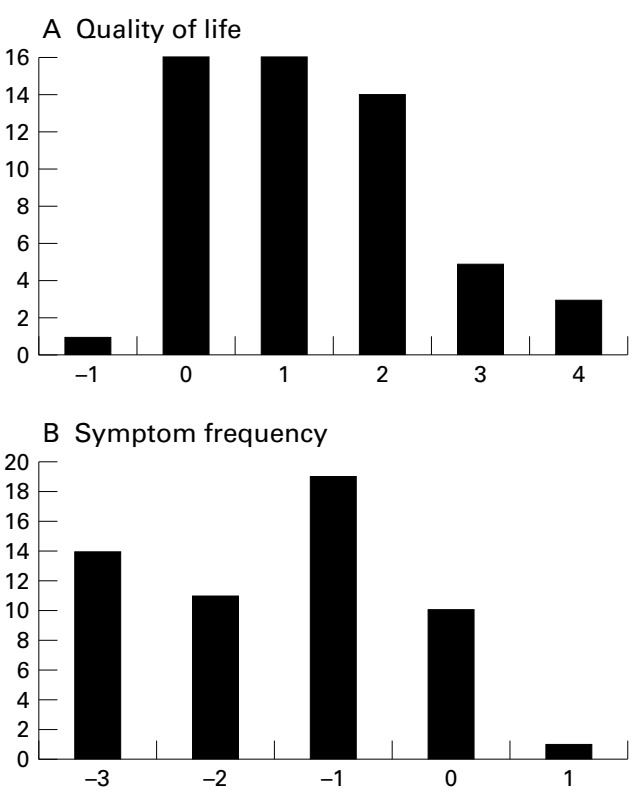

C Symptom severity

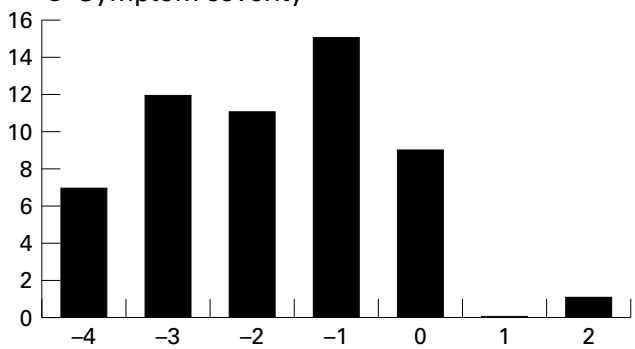

Figure 1 Changes in each patient's scores following ablation. Quality of life, symptom frequency, and symptom severity score changes are shown. An improvement in quality of life is denoted by positive values $(A)$; a reduction in symptom frequency and severity following ablation is denoted by negative values $(B, C)$. The time interval following ablation is compared with a similar time interval before ablation.

quality of life score and a reduction in both symptom frequency and symptom severity scores $(\mathrm{p}<0.001)$.

The change in each patient's quality of life, symptom frequency score, and symptom severity score is shown in fig 1 . Of the 55 patients, only one $(1.8 \%)$ reported a deterioration in quality of life. This patient had both atrial fibrillation and atrial flutter before ablation, which were refractory to medical treatment including amiodarone. Following ablation the patient continued to experience symptomatic paroxysmal atrial fibrillation. Thirty eight patients $(69 \%)$ had an improvement and 16 $(29 \%)$ had no improvement in quality of life score following ablation (fig 1A). Thirteen of the latter 16 patients scored their preablation quality of life as good to excellent despite the fact that six of them reported their symptoms as either very frequent or severe. The overall improvement in quality of life was assessed by a second question in which patients assigned a score to their general quality of life since their ablation. The mean (SEM) for the group was $4.2(0.1)$, compared with an expected score of 3.0 if quality of life was unchanged.

The majority of patients had a reduction in symptom frequency $(80 \%)$ and in symptom severity $(82 \%)$, as shown in fig $1 \mathrm{~B}$ and $1 \mathrm{C}$. The same patient who reported a reduction in quality of life was the only patient to report an increase in symptom frequency and severity. Only three of the 10 patients who reported no change in symptom frequency scored their symptoms as very frequent before ablation, and only three of nine patients who reported no change in symptom severity scored their symptoms as severe or very severe before ablation.

The results of the patient questionnaire were analysed according to the presence or absence of atrial fibrillation before ablation (table 4). The atrial fibrillation group had a significant improvement in quality of life and a reduction in symptom frequency and severity $(\mathrm{p}<0.001)$. Patients with atrial flutter alone (no atrial fibrillation) reported a greater improvement in their quality of life and a greater reduction in symptom frequency and severity than the atrial fibrillation group, but changes did not reach significance except possibly for the symptom severity scores $(p=0.03)$.

\section{Discussion}

Our study of a consecutive series of patients showed that atrial flutter ablation resulted in a substantial improvement in quality of life, a reduction in symptom frequency and symptom severity, and less antiarrhythmic drug and hospital resource utilisation. The clinical benefit most probably reflected a reduction in arrhythmia burden, as evidenced by the changes in symptom frequency and severity scores, and perhaps also a reduction in antiarrhythmic drug requirements. In common with other series in which bidirectional isthmus conduction block is the end point for ablation, catheter ablation was performed with a high success rate, a low complication rate, and a low rate of atrial flutter recurrence on long term follow up. ${ }^{4}$ Allowing for the fact that four patients with symptomatic atrial fibrillation progressed to an ablate and pace procedure and were excluded, it is encouraging that, of 29 patients with both atrial fibrillation and atrial flutter before

Table 4 Questionnaire results according to the presence or absence of atrial fibrillation before ablation

\begin{tabular}{|c|c|c|c|c|c|c|}
\hline & \multicolumn{3}{|c|}{ No atrial fibrillation } & \multicolumn{3}{|c|}{ Atrial fibrillation } \\
\hline & Preablation & Follow up & $\triangle$ Score & Preablation & Follow up & $\Delta$ Score \\
\hline Quality of life score & $2.5(0.2)$ & $4.1(0.2)^{\star}$ & $+1.6(0.2)$ & $2.5(0.2)$ & $3.5(0.2)^{\star}$ & $+1.0(0.2) \dagger$ \\
\hline Symptom frequency score & $3.4(0.2)$ & $1.7(0.2)^{\star}$ & $-1.8(0.2)$ & $3.5(0.1)$ & $2.3(0.2)^{\star}$ & $-1.2(0.2) \dagger$ \\
\hline Symptom severity score & $3.9(0.2)$ & $1.7(0.2)^{\star}$ & $-2.3(0.3)$ & $3.7(0.2)$ & $2.2(0.2)^{\star}$ & $-1.5(0.3) \ddagger$ \\
\hline
\end{tabular}

Result are mean (SEM); $\Delta$ score, change in score comparing follow up with a similar time interval preablation.

${ }^{\star} \mathrm{p}<0.001 v$ preablation scores (sign test).

+NS $v \Delta$ score in the no atrial fibrillation group (Mann-Whitney U test).

$\neq \mathrm{p}=0.03 v \Delta$ score in the no atrial fibrillation group (Mann-Whitney U test). 
ablation, only one reported a deterioration in quality of life and symptoms. Compared with other published reports our study followed a consecutive series of patients rather than just the subgroup with bidirectional isthmus conduction block, and clearly showed the change in scores in each patient in addition to the overall changes for the group..$^{11}$ Natale and colleagues conducted a prospective randomised comparison of antiarrhythmic drug treatment versus first line radiofrequency ablation in a select group of patients with atrial flutter. ${ }^{9}$ Patients with prior evidence of atrial fibrillation, left atrial enlargement, or previous antiarrhythmic drug failure were excluded. Quality of life improved significantly in the catheter ablation group and changed little in the drug treatment group, supporting the use of catheter ablation as first line treatment in patients with atrial flutter as their only presenting atrial tachyarrhythmia. The results of our study suggest that patients who are less rigorously selected may also benefit from atrial flutter ablation.

\section{ATRIAL FIBRILLATION}

Patients with both atrial flutter and atrial fibrillation before ablation had a significant improvement in quality of life and reduction in symptom frequency and severity following catheter ablation of their atrial flutter. During a mean follow up period of one year, less than half the atrial fibrillation group had a documented recurrence of atrial fibrillation. Other investigators have reported similar reductions in atrial fibrillation following atrial flutter ablation. ${ }^{113-15}$ This supports the concept that atrial flutter helps to maintain atrial fibrillation in a proportion of these patients, resulting in sustained (and documented) atrial fibrillation; atrial flutter ablation may result in a normalisation of atrial refractoriness and reversal of electrical remodelling, making atrial fibrillation less likely. ${ }^{16}{ }^{17}$ Our results also support the hypothesis that atrial fibrillation-in at least some patients with atrial flutter-is maintained by re-entry around the tricuspid valve orifice. ${ }^{1819}$ According to this hypothesis reentry around a stable anatomical pathway such as the tricuspid annulus might serve as a back up mechanism maintaining atrial fibrillation. ${ }^{20}$ We therefore recommend atrial flutter ablation for patients with both atrial flutter and atrial fibrillation on the grounds that the cure rate for atrial flutter is high and catheter ablation has a positive impact on the recurrence and possibly on the severity of atrial fibrillation. Baseline characteristics of the four patients who proceeded to atrioventricular node ablation and pacemaker implantation were similar to the remaining 55 patients in this study, and in retrospect these patients were not identifiable in advance.

LIMITATIONS

This study may have been influenced by recall bias, as patients were asked to compare the time interval following ablation with a similar time interval before ablation. However the significant reduction in antiarrhythmic drug use and hospital resource utilisation, as confirmed by a review of the medical records, suggests that recall bias is unlikely to have altered the results substantially. Despite the fact that Holter recordings and event monitors were available, it is likely that patients experienced episodes of paroxysmal atrial fibrillation during the follow up period which were not documented. This is suggested by the fact that $47 \%$ of patients reported experiencing an attack of their rhythm problem following ablation, whereas atrial fibrillation was documented in only $28 \%$ during follow up. It is possible, as discussed above, that re-entry around a stable anatomical pathway such as the tricuspid annulus might serve as a back up mechanism maintaining atrial fibrillation and, following atrial flutter ablation, atrial fibrillation may be paroxysmal rather than persistent during follow up. This requires further investigation. Formal cost effectiveness analysis was not performed in this study. However, the substantial reduction in hospital resource utilisation following ablation strongly argues in favour of the cost effectiveness of atrial flutter ablation.

\section{CONCLUSIONS}

Atrial flutter ablation resulted in significant symptomatic improvements, both in patients with atrial flutter alone and in those with concomitant atrial fibrillation. Atrial flutter ablation has a positive impact on the recurrence of atrial fibrillation. These results suggest that a prospective randomised trial of catheter ablation versus antiarrhythmic drug treatment in patients with both atrial flutter and atrial fibrillation would be worthwhile.

1 Olgin JE, Kalman JM, Lesh MD. Conduction barriers in human atrial flutter: correlation of electrophysiology and anatomy. $\mathcal{F}$ Cardiovasc Electrophysiol 1996;7:1112-26.

2 Cosio FG, Lopez-Giol M, Goicolea A, et al. Radiofrequency ablation of the inferior vena cava-tricuspid valve isthmus in common atrial flutter. Am $\mathcal{f}$ Cardiol 1993;71:705-9.

3 Cabrera JA, Sanchez-Quintana D, Ho SY, et al. Angiographic anatomy of the inferior right atrial isthmus in patients with and without history of common atrial flutter. Circulation 1999;99:3017-23.

4 Schwartzman D, Callans DJ, Gottlier CD, et al. Conduction block in the inferior vena caval-trisuspid valve isthmus: association with outcome of radiofrequency ablation of type I atrial flutter. F Am Coll Cardiol 1996;28:1519-31.

5 Schumacher B, Pfeiffer D, Tebbenjohanns J, et al. Acute and long-term effects of consecutive radiofrequency applications on conduction properties of the subeustachian isthmus in type I atrial flutter. $\mathcal{F}$ Cardiovasc Electrophysiol 1998;9:152-63.

6 Iesaka Y, Takahashi A, Goya M, et al. High energy radiofrequency catheter ablation for common atrial flutter targeting the isthmus between the inferior vena cava and tricuspid valve annulus using a super long tip electrode. Pacing Clin Electrophysiol 1998;21:401-9.

7 Cauchemez B, Haissaguerre M, Fischer B, et al. Electrophysiological effects of catheter ablation of inferior vena cava-tricuspid annulus isthmus in common atrial flutter. Circulation 1996;93:284-94.

8 Poty H, Saoudi N, Abdel Aziz A, et al. Radiofrequency catheter ablation of type I atrial flutter. Prediction of late success by electrophysiological criteria. Circulation 1995; 92:1389-92

9 Natale A, Newby KH, Pisano E, et al. Prospective randomized comparison of antiarrhythmic therapy versus first-line radiofrequency ablation in patients with atrial flutter. F Am Coll Cardiol 2000;35:1898-904.

10 Anselme F, Saoudi N, Poty H, et al. Radiofrequency catheter ablation of common atrial flutter: significance of palpitations and quality-of-life evaluation in patien
isthmus block. Circulation 1999:99:534-40.

11 Lee SH, Tai CT, Yu WC, et al. Effects of radiofrequency catheter ablation on quality of life in patients with atrial flutter. Am $\mathcal{F}$ Cardiol 1999;84:278-83. 
12 Fitzpatrick AP, Kourouyan HD, Siu A, et al. Quality of life and outcomes after radiofrequency His-bundle catheter ablation and permanent pacemaker implantation: impact of treatment in paroxysmal and established atrial fibrillation. Am Heart f 1996;131:499-507.

13 Movsowitz C, Callans DJ, Schwartzman D, et al. The results of atrial flutter ablation in patients with and without a history of atrial fibrillation. Am F Cardiol 1996;78:93-6.

14 Tai CT, Chen SA, Chiang CE, et al. Long-term outcome of radiofrequency catheter ablation for typical atrial flutter risk prediction of recurrent arrhythmias. $\mathcal{f}$ Cardiovasc Electrophysiol 1998;9:115-21.

15 Katritsis D, Iliodromitis E, Fragakis N, et al. Ablation therapy of type I atrial flutter may eradicate paroxysmal atrial fibrillation. Am f Cardiol 1996;78:345-7.

16 Roithinger FX, Lesh MD. What is the relationship of atrial flutter and fibrillation? Pacing Clin Electrophysiol 1999;22: 643-54.
17 Cox JL, Canavan TE, Schuessler RB, et al. The surgical treatment of atrial fibrillation: II: Intraoperative electrophysiologic mapping and description of the electrophysiologic basis of atrial flutter and atrial fibrillation. $\mathcal{F}$ Thorac Cardiovasc Surg.1991;101:406-26.

18 Nabar A, Rodriguez LM, Timmermans C, et al. Effect of right atrial isthmus ablation on the occurrence of atrial fibrillation. Observations in four patient groups having type I atrial flutter with or without associated atrial fibrill type I atrial flutter with or without a 99:1441-5.

19 Kumagai K, Khrestian C, Waldo AL. Simultaneous multisite mapping studies during induced atrial fibrillation in the sterile pericarditis model: insights into the mechanism of its maintenance. Circulation 1997;95:51121.

20 Allessis MA. Atrial electrophysiologic remodeling: another vicious circle? f Cardiovasc Electrophysiol 1998;9:1378-93.

\section{IMAGES IN CARDIOLOGY}

\section{Influence of slow coronary blood flow on stenosis morphology}

A 62 year old man was admitted because of frequent episodes of palpitation and chest pain. Two dimensional echocardiography showed hypokinesis of the posterolateral wall with preserved global left ventricular function and volumes. Holter monitoring showed several episodes of non-sustained ventricular tachycardia, and the subsequent electrophysiologic study confirmed the presence of non-sustained inducible ventricular tachycardia. Coronary angiography showed slow coronary run-off of contrast medium and a complex eccentric lesion in the proximal portion of the left circumflex coronary artery (1). The intracoronary injection of glyceryl trinitrate $(200 \mu \mathrm{g})$ caused diffuse coronary vasodilation but it
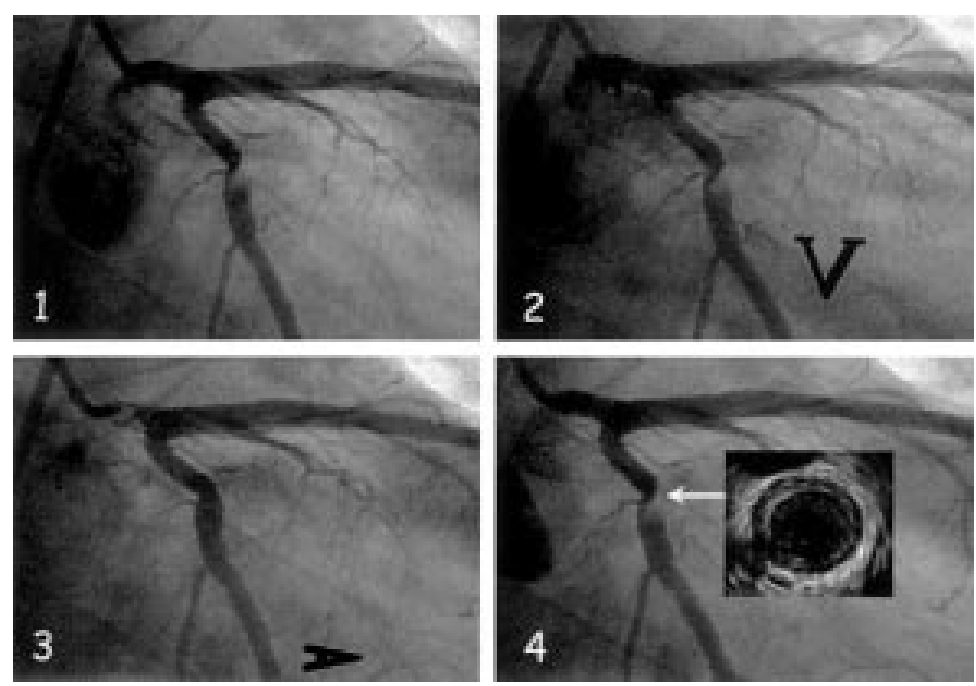

failed to affect both stenosis severity and morphology and contrast medium run-off (2). Conversely, intracoronary injection of adenosine $(20 \mu \mathrm{g})$ was followed by normalisation of contrast medium run-off and a striking modification of stenosis morphology revealing a mild concentric plaque (3). Soon after adenosine was washed out, the same complex eccentric lesion reappeared (4). However, intracoronary ultrasound confirmed the presence of a mild concentric fibrous plaque only (arrow indicates the site to which the ultrasound image refers).

Slow coronary run-off caused by coronary microcirculation dysfunction might be responsible for flow turbulence causing inhomogeneous contrast medium distribution at the site of mild plaques; this, in turn, can simulate the presence of a severe complex stenosis. Accordingly, in our patient glyceryl trinitrate, which causes epicardial coronary artery dilation but only mild microvascular dilation, probably failed to increase coronary blood flow velocity and to affect the distribution of contrast medium at the site of the plaque. By contrast, adenosine, a potent vasodilator of the coronary microcirculation, increased coronary flow velocity, thus unmasking the artefact caused by contrast medium turbulence. In patients with slow coronary run-off and apparently complex lesions, intracoronary injection of adenosine may be useful in order to avoid inappropriate revascularisation procedures.

FABRIZIO TOMAI ANNA S GHINI A DANESI f.tomai@tiscalinet.it 